\title{
Energy transfer in open quantum systems weakly coupled with two reservoirs
}

\author{
Franco FAGNOLA ${ }^{1}$ \\ Damiano Polettio ${ }^{2}$ \\ EMANUEla SASSO 3 \\ 1,2 Dipartimento di Matematica, \\ Politecnico di Milano, Piazza Leonardo \\ da Vinci 32, I-20133 Milano, Italy. \\ franco.fagnola@polimi.it; \\ damiano.poletti@polimi.it \\ 3 Dipartimento di Matematica, \\ Università di Genova, Via Dodecaneso \\ 35, I - 16146 Genova, Italy. \\ sasso@dima.unige.it
}

\begin{abstract}
We show that the energy transfer through an open quantum system with non-degenerate Hamiltonian weakly coupled with two reservoirs in equilibrium is approximately proportional to the difference of their temperatures unless both temperatures are small.
\end{abstract}

\section{RESUMEN}

Mostramos que la transferencia de energía a través de un sistema cuántico abierto con Hamiltoniano no-degenerado débilmente acoplado con dos reservorios en equilibrio es aproximadamente proporcional a la diferencia de sus temperaturas a menos que ambas temperaturas sean pequeñas.

Keywords and Phrases: weak-coupling, quantum Markov semigroup, quantum transport, energy current.

2020 AMS Mathematics Subject Classification: 81S22, 82C10, 80A19. 


\section{Introduction}

Energy transfer in classical and quantum systems and the validity of Fourier's law of heat conduction have been a hot topic for many years (see [3, 4, 6, 7, 12, 18, 25, 26] and the references therein). For quantum systems, in particular, after experimental evidence of effective quantum energy transfer in photosynthesis in some biological systems has been found (see [14, 24]), investigations have focused on understanding to what extent quantum mechanics contributes to transport efficiency.

Several models have been proposed involving open quantum systems (see e.g. [5, 6, 27]), mostly phenomenological, and also numerical simulations have been done showing different behaviours. The interaction of the open quantum system with reservoirs is described through interaction operators that appear in the dissipative part of the Gorini-Kossakowski-Sudharshan-Lindblad (GKSL) $[17,22]$ generator $\mathcal{L}$ of the dynamics, while the Hamiltonian part is given by the commutator with the system Hamiltonian $H_{S}$. However, when the GKSL generator is rigorously deduced from some scaling (weak coupling or low density limit) both the system Hamiltonian and the interaction operators appear in the GKSL generator $\mathcal{L}$ after non-trivial transformations (see $[1,2,9,10,13,19]$ ).

In this paper we study models of open quantum systems rigorously deduced from the weak coupling limit. We consider a quantum system with non-degenerate Hamiltonian $H_{S}$ coupled with two reservoirs in equilibrium at inverse temperatures $\beta_{1} \leq \beta_{2}$ and study variation of energy due to couplings with each reservoir. It is well-known (see Lebowitz and Spohn [25] (V.28)) that, by the second law of thermodynamics, energy (heat) flows from the hotter to the cooler reservoir. The energy flow, in general, is not proportional to the difference of temperature because of the nonlinear dependence of susceptibilities on temperature, namely an exact Fourier's law does not hold.

However, we rigorously prove that it holds in an approximate way when the temperatures of reservoirs are not too small or, as an alternative, differences between nearest energy levels are small. More precisely, we show that the amount of energy flowing through the system, Theorem 4.2 , formula (4.5), is approximately proportional to the product of the temperature differences and a constant (conductivity) which can be interpreted as the average energy needed to jump from a level to the following higher level.

The paper is organised as follows. In Section 2 we introduce quantum Markov semigroups (QMS) arising from the weak coupling limit of a non-degenerate system with two Boson reservoirs. The energy flow is computed explicitly in Section 3, Theorem 3.3, formula (3.7). The dependence of the energy flow on temperatures is studied in Section 4. Moreover, we also study (Theorem 4.3) the asymptotic behaviour of the invariant state when the eigenvalues of $H_{S}$ increase in number and form a set more and more packed. It turns out that the invariant state converges towards a 
Gibbs state with temperature equal to the mean temperatures of the two baths.

Finally, in Section 5, we consider as system the Ising model Hamiltonian and show that the energy flow in this case is zero. We have not been able to extend our analysis to quantum spin chains because their Hamiltonians are highly degenerate and the GKSL generator arising from the weak coupling limit, albeit explicit, is not easily treatable. In particular, we could not extract the relevant information on invariant states.

\section{Semigroups of weak coupling limit type}

We consider an open quantum system with Hamiltonian $H_{S}$ acting on a complex separable Hilbert space $\mathrm{h}$ with discrete spectral decomposition

$$
H_{S}=\sum_{m \geq 0} \varepsilon_{m} P_{\varepsilon_{m}}
$$

where $\varepsilon_{m}$, with $\varepsilon_{m}<\varepsilon_{n}$ for $m<n$, are the eigenvalues of $H_{S}$ and $P_{\varepsilon_{m}}$ are the corresponding eigenprojectors. The system is coupled with two reservoirs each one in equilibrium with inverse temperatures $\beta_{1} \leq \beta_{2}$ with interaction Hamiltonians

$$
H_{1}=D_{1} \otimes A^{+}\left(\phi_{1}\right)+D_{1}^{*} \otimes A^{-}\left(\phi_{1}\right), \quad H_{2}=D_{2} \otimes A^{+}\left(\phi_{2}\right)+D_{2}^{*} \otimes A^{-}\left(\phi_{2}\right),
$$

where $D_{1}, D_{2}$ are bounded operators on $\mathrm{h}$ and $A^{+}\left(\phi_{j}\right), A^{-}\left(\phi_{j}\right)$ creation and annihilation operators, in the Fock space of the reservoir $j$, with test function $\phi_{j}$.

It is well-known (see $[2,9,13,25])$ that, in the weak coupling limit, the evolution of the system observables is governed by a quantum Markov semigroup (QMS) on $\mathcal{B}(\mathrm{h})$, the algebra of all bounded operators in $\mathrm{h}$, with generator of the form

$$
\mathcal{L}=\sum_{j=1,2, \omega \in \mathrm{B}} \mathcal{L}_{j, \omega}
$$

where $B$ is the set of all Bohr frequencies

$$
\mathrm{B}:=\left\{\omega \mid \exists \varepsilon_{n}, \varepsilon_{m} \text { s.t. } \omega=\varepsilon_{n}-\varepsilon_{m}>0\right\} .
$$

For every Bohr frequency $\omega, \mathcal{L}_{j, \omega}$ is a generator with the Gorini-Kossakowski-Sudharshan-Lindblad (GKSL) structure (see [17, 22])

$$
\begin{aligned}
\mathcal{L}_{j, \omega}(x) & =\mathrm{i}\left[H_{j, \omega}, x\right]-\frac{\Gamma_{j, \omega}^{-}}{2}\left(D_{j, \omega}^{*} D_{j, \omega} x-2 D_{j, \omega}^{*} x D_{j, \omega}+x D_{j, \omega} D_{j, \omega}^{*}\right) \\
& -\frac{\Gamma_{j, \omega}^{+}}{2}\left(D_{j, \omega} D_{j, \omega}^{*} x-2 D_{j, \omega} x D_{j, \omega}^{*}+x D_{j, \omega} D_{j, \omega}^{*}\right)
\end{aligned}
$$

for all $x \in \mathcal{B}(\mathrm{h})$, with Kraus operators $D_{j, \omega}$ defined by

$$
D_{j, \omega}=\sum_{\left(\varepsilon_{n}, \varepsilon_{m}\right) \in \mathrm{B}_{\omega}} P_{\varepsilon_{m}} D_{j} P_{\varepsilon_{n}}
$$


where $\mathrm{B}_{\omega}=\left\{\left(\varepsilon_{n}, \varepsilon_{m}\right) \mid \varepsilon_{n}-\varepsilon_{m}=\omega\right\}, \Gamma_{j, \omega}^{ \pm}=f_{j, \omega} \gamma_{j, \omega}^{ \pm}$

$$
\gamma_{j, \omega}^{-}=\frac{\mathrm{e}^{\beta_{j} \omega}}{\mathrm{e}^{\beta_{j} \omega}-1}, \quad \gamma_{j, \omega}^{+}=\frac{1}{\mathrm{e}^{\beta_{j} \omega}-1}, \quad f_{j, \omega}=\int_{\left\{y \in \mathbb{R}^{3}|| y \mid=\omega\right\}}\left|\phi_{j}(y)\right|^{2} \mathrm{~d}_{s} y
$$

( $\mathrm{d}_{s}$ denotes the surface integral) and $H_{j, \omega}$ are bounded self-adjoint operators on $\mathrm{h}$ commuting with $H_{S}$ of the form

$$
H_{j, \omega}=\kappa_{j, \omega}^{-} D_{j, \omega}^{*} D_{j, \omega}+\kappa_{j, \omega}^{+} D_{j, \omega} D_{j, \omega}^{*}
$$

for some real constants $\kappa_{j, \omega}^{ \pm}$.

In the sequel, following a customary convention to simplify the notation, we also denote $D_{j, \omega}^{-}:=D_{j, \omega}$ and $D_{j, \omega}^{+}:=D_{j, \omega}^{*}$ and write

$$
\mathcal{Q}_{j, \omega}^{ \pm}(x)=-\frac{1}{2} D_{j, \omega}^{\mp} D_{j, \omega}^{ \pm} x+D_{j, \omega}^{\mp} x D_{j, \omega}^{ \pm}-\frac{1}{2} x D_{j, \omega}^{\mp} D_{j, \omega}^{ \pm}
$$

the term of the GKSL generator arising from the interaction with the bath $j$ due the Bohr frequency $\omega$ is

$$
\mathcal{L}_{j, \omega}=\Gamma_{j, \omega}^{-} \mathcal{Q}_{j, \omega}^{-}+\Gamma_{j, \omega}^{+} \mathcal{Q}_{j, \omega}^{+}+\mathrm{i}\left[H_{j, \omega}, \cdot\right]
$$

and the term arising from the interaction with the reservoir $j$ is

$$
\mathcal{L}_{j}=\sum_{\omega \in \mathrm{B}} \mathcal{L}_{j, \omega}
$$

We now make some assumptions on constants in such a way as to ensure boundedness of operators $\mathcal{L}_{j}$. First of all note that the series $\sum_{\omega} D_{j, \omega}^{*} D_{j, \omega}$ is strongly convergent. Indeed, for all vector $u=\sum_{n \geq 0} P_{\varepsilon_{n}} u$ in $\mathrm{h}$, we have

$$
\begin{aligned}
\sum_{\omega}\left\langle u, D_{j, \omega}^{*} D_{j, \omega} u\right\rangle & =\sum_{\omega} \sum_{n, m \geq 0}\left\langle P_{\varepsilon_{m}-\omega} D_{j} P_{\varepsilon_{m}} u, P_{\varepsilon_{n}-\omega} D_{j} P_{\varepsilon_{n}} u\right\rangle \\
& =\sum_{\omega} \sum_{n \geq 0}\left\langle D_{j} P_{\varepsilon_{n}} u, P_{\varepsilon_{n}-\omega} D_{j} P_{\varepsilon_{n}} u\right\rangle \\
& \leq \sum_{n \geq 0}\left\|D_{j} P_{\varepsilon_{n}} u\right\|^{2} \\
& =\left\|D_{j}\right\|^{2}\|u\|^{2} .
\end{aligned}
$$

As a consequence, if we assume

$$
\sup _{\omega \in \mathrm{B}} \Gamma_{j, \omega}^{ \pm}<+\infty, \quad \sup _{\omega \in \mathrm{B}}\left|\kappa_{j, \omega}^{ \pm}\right|<+\infty,
$$

for $j=1,2$ GKSL generators $\mathcal{L}_{j}$ turn out to be bounded. The above condition will be assumed to be in force throughout the paper.

Remark. Note that $\mathcal{L}_{j}$ depends on the inverse temperature $\beta_{j}$ only through the constants $\gamma_{j, \omega}^{ \pm}$. The above notation follows that of [1]. 
For all normal linear operator $\mathcal{S}$ on $\mathcal{B}(\mathrm{h})$ we denote by $\mathcal{S}_{*}$ the predual operator acting on the Banach space of trace class operators on $\mathrm{h}$. Therefore, we denote by $\mathcal{T}=\left(\mathcal{T}_{t}\right)_{t \geq 0}$ the QMS on $\mathcal{B}(\mathrm{h})$ generated by $\mathcal{L}$ and by $\mathcal{T}_{*}=\left(\mathcal{T}_{* t}\right)_{t \geq 0}$ the predual semigroup acting on trace class operators. In the same way, $\mathcal{T}^{j}$ (resp. $\mathcal{T}^{j, \omega}$ and $\mathcal{T}_{*}^{j, \omega}$ ) stand for the QMS generated by $\mathcal{L}_{j}$ (resp. $\mathcal{L}_{j, \omega}$ and its predual semigroup). In this paper we are concerned with normal states, therefore we shall identify them with their densities which are positive operators on $\mathrm{h}$ with unit trace.

We end this section by checking that, if reservoirs have the same temperature $\beta_{1}=\beta_{2}=\beta$ and $Z_{\beta}:=\operatorname{tr}\left(\mathrm{e}^{-\beta H_{S}}\right)<+\infty$, then the Gibbs state has density

$$
\rho_{\beta}=Z_{\beta}^{-1} \mathrm{e}^{-\beta H_{S}}
$$

and is stationary.

Proposition 2.1. If $\beta_{1}=\beta_{2}=\beta$ and

$$
Z_{\beta}:=\operatorname{tr}\left(\mathrm{e}^{-\beta H_{S}}\right)=\sum_{n \geq 0} e^{-\beta \varepsilon_{n}} \operatorname{dim}\left(P_{\varepsilon_{n}}\right)<+\infty
$$

then the Gibbs state (2.7) is invariant for all QMSs generated by $\mathcal{L}, \mathcal{L}_{1}, \mathcal{L}_{2}$.

Proof. We begin by observing that for $\left(\varepsilon_{n}+\omega, \varepsilon_{n}\right),\left(\varepsilon_{n}, \varepsilon_{n}-\omega\right) \in \mathrm{B}_{\omega}$, we can compute directly

$$
\begin{aligned}
\left(\mathcal{L}_{j, \omega}\right)_{*}\left(P_{\varepsilon_{n}}\right)= & \Gamma_{j, \omega}^{-}\left(P_{\varepsilon_{n}-\omega} D_{j} P_{\varepsilon_{n}} D_{j}^{*} P_{\varepsilon_{n}-\omega}-P_{\varepsilon_{n}} D_{i}^{*} P_{\varepsilon_{n}-\omega} D_{j} P_{\varepsilon_{n}}\right)+ \\
& \Gamma_{j, \omega}^{+}\left(P_{\varepsilon_{n}+\omega} D_{j}^{*} P_{\varepsilon_{n}} D_{j} P_{\varepsilon_{n}+\omega}-P_{\varepsilon_{n}} D_{j} P_{\varepsilon_{n}+\omega} D_{j}^{*} P_{\varepsilon_{n}}\right) .
\end{aligned}
$$

A state of the form $\rho=\sum_{n} \rho_{\varepsilon_{n}} P_{\varepsilon_{n}}$, which is a function of the system Hamiltonian $H_{S}$ (also called a diagonal state), satisfies

$$
\begin{aligned}
\mathcal{L}_{* j}(\rho)= & \sum_{\omega} \sum_{n}\left(\mathcal{L}_{j, \omega}\right)_{*}\left(\rho_{\varepsilon_{n}} P_{\varepsilon_{n}}\right) \\
= & \sum_{\omega} \sum_{\left(\varepsilon_{n}+\omega, \varepsilon_{n}\right) \in \mathrm{B}_{\omega}}\left(\rho_{\varepsilon_{n}+\omega} \Gamma_{j, \omega}^{-}-\rho_{\varepsilon_{n}} \Gamma_{j, \omega}^{+}\right) P_{\varepsilon_{n}} D_{j} P_{\varepsilon_{n}+\omega} D_{j}^{*} P_{\varepsilon_{n}}+ \\
& \sum_{\omega} \sum_{\left(\varepsilon_{n}, \varepsilon_{n}-\omega\right) \in \mathrm{B}_{\omega}}\left(\rho_{\varepsilon_{n}-\omega} \Gamma_{j, \omega}^{+}-\rho_{\varepsilon_{n}} \Gamma_{j, \omega}^{-}\right) P_{\varepsilon_{n}} D_{j}^{*} P_{\varepsilon_{n}-\omega} D_{j} P_{\varepsilon_{n}} .
\end{aligned}
$$

Now if $\beta_{1}=\beta_{2}=\beta$ and $\rho_{\varepsilon_{n}}=\mathrm{e}^{-\beta \varepsilon_{n}}$ as in (2.7), we have

$$
\frac{\Gamma_{j, \omega}^{+}}{\Gamma_{j, \omega}^{-}}=\frac{\gamma_{j, \omega}^{+}}{\gamma_{j, \omega}^{-}}=e^{-\beta \omega}=\frac{\rho_{\varepsilon_{n}+\omega}}{\rho_{\varepsilon_{n}}},
$$

for all $j=1,2$, so that $\mathcal{L}_{* j}(\rho)=0$ and $\rho=\mathrm{e}^{-\beta H_{S}} / Z_{\beta}$ is an invariant state for the QMS generated by $\mathcal{L}_{j}$. Since $\mathcal{L}=\mathcal{L}_{1}+\mathcal{L}_{2}$ it is an invariant state also for the QMS generated by $\mathcal{L}$. 


\section{$3 \quad$ Energy current}

The rate of energy variation in the system, in a state $\rho$, due to interaction with the reservoir $j$ is $\operatorname{tr}\left(\rho \mathcal{L}_{j}\left(H_{S}\right)\right)$ (see [25] (V.28)). Therefore

$$
\operatorname{tr}\left(\rho \mathcal{L}_{1}\left(H_{S}\right)\right)-\operatorname{tr}\left(\rho \mathcal{L}_{2}\left(H_{S}\right)\right)
$$

is twice the rate at which the energy flows through the system from the hotter bath to the colder bath, namely, the energy current through the system.

Adapting a result by Lebowitz and Spohn [25] Theorem 2 and Corollary 1, it is possible to prove that the energy current is non-negative for finite dimensional systems.

Theorem 3.1. Suppose that $\mathrm{h}$ is finite dimensional and let $\rho$ be a faithful invariant state, then the energy current (3.1) is non-negative.

Proof. If a system is weakly coupled to a single bath $j$ at inverse temperature $\beta_{j}$, it is well-known that the Gibbs state $\rho_{\beta_{j}}=Z_{\beta_{j}}^{-1} \mathrm{e}^{-\beta_{j} H_{S}}$, with $Z_{\beta_{j}}=\operatorname{tr}\left(\mathrm{e}^{-\beta_{j} H_{S}}\right)$, is invariant.

Consider the relative entropy of $\rho$ with respect to $\rho_{\beta_{j}}$ defined by $S\left(\rho \mid \rho_{\beta_{j}}\right)=\operatorname{tr}\left(\rho\left(\log \left(\rho-\log \rho_{\beta_{j}}\right)\right)\right.$ which is a notoriously non-increasing function (see [23], Theorem 1.5), i.e.

$$
S\left(\mathcal{T}_{* t}^{j}(\rho) \mid \mathcal{T}_{* t}^{j}\left(\rho_{\beta_{j}}\right)\right) \leq S\left(\rho \mid \rho_{\beta_{j}}\right),
$$

for all $\rho$ and $t \geq 0$. States $\mathcal{T}_{* t}^{j}(\rho), j=1,2$ will still be faithful for small $t$, therefore no problem arises when considering logarithms. Since $\rho_{\beta_{j}}$ is invariant, denoting $\rho_{t}:=\mathcal{T}_{* t}^{j}(\rho)$, and differentiating we find

$$
\begin{aligned}
\frac{\mathrm{d}}{\mathrm{d} t} S\left(\rho_{t} \mid \rho_{\beta_{j}}\right) & =\frac{\mathrm{d}}{\mathrm{d} t} \operatorname{tr}\left(\rho_{t}\left(\log \rho_{t}-\log \rho_{\beta_{j}}\right)\right) \\
& =\operatorname{tr}\left(\rho_{t}^{\prime}\left(\log \rho_{t}-\log \rho_{\beta_{j}}\right)\right)+\operatorname{tr}\left(\rho_{t} \frac{\mathrm{d}}{\mathrm{d} t} \log \rho_{t}\right) .
\end{aligned}
$$

Since for every $x>0, \log x=\int_{0}^{+\infty}\left(\frac{1}{1+s}-\frac{1}{x+s}\right) \mathrm{d} s$,

$$
\frac{\mathrm{d}}{\mathrm{d} t} \log \rho_{t}=\int_{0}^{+\infty}\left(s+\rho_{t}\right)^{-1} \rho_{t}^{\prime}\left(s+\rho_{t}\right)^{-1} \mathrm{~d} s
$$

so that

$$
\operatorname{tr}\left(\rho_{t} \frac{\mathrm{d}}{\mathrm{d} t} \log \rho_{t}\right)=\operatorname{tr}\left(\rho_{t}^{\prime} \int_{0}^{+\infty} \rho_{t}\left(s+\rho_{t}\right)^{-2} \mathrm{~d} s\right)=\operatorname{tr}\left(\rho_{t}^{\prime}\right)=0 .
$$

By imposing $\rho_{\beta_{j}}=Z_{\beta_{j}}^{-1} e^{-\beta_{j} H_{S}}$, and recalling that $\rho_{t}^{\prime}=\mathcal{L}_{* j}\left(\rho_{t}\right), \operatorname{tr}\left(\rho_{t}^{\prime}\right)=0$ by trace preservation, we obtain

$$
\begin{aligned}
\frac{\mathrm{d}}{\mathrm{d} t} S\left(\rho_{t} \mid \rho_{\beta_{j}}\right) & =\operatorname{tr}\left(\rho_{t}^{\prime}\left(\log \rho_{t}-\log \rho_{\beta_{j}}\right)\right) \\
& =\operatorname{tr}\left(\rho_{t}^{\prime}\left(\log \rho_{t}+\beta_{j} H_{S}-\log Z_{\beta_{j}}^{-1}\right)\right) \\
& =\operatorname{tr}\left(\rho_{t}^{\prime} \log \rho_{t}\right)+\beta_{j} \operatorname{tr}\left(\rho_{t} \mathcal{L}_{j}\left(H_{S}\right)\right) .
\end{aligned}
$$


In particular $\operatorname{tr}\left(\rho_{t}^{\prime}\left(\log \rho_{t}\right)\right)+\beta_{j} \operatorname{tr}\left(\rho_{t} \mathcal{L}\left(H_{S}\right)\right) \leq 0$ by monotonicity of the relative entropy, namely

$$
-\operatorname{tr}\left(\mathcal{L}_{* j}\left(\rho_{t}\right) \log \rho_{t}\right)-\beta_{j} \operatorname{tr}\left(\rho_{t} \mathcal{L}_{j}\left(H_{S}\right)\right) \geq 0 .
$$

In our context, the entropy production of the system due to interaction with the bath at inverse temperature $\beta_{j}$ is

$$
-\operatorname{tr}\left(\mathcal{L}_{* j}\left(\rho_{t}\right) \log \rho_{t}\right)-\beta_{j} \operatorname{tr}\left(\rho_{t} \mathcal{L}_{j}\left(H_{S}\right)\right) \geq 0 .
$$

Now, for all $\beta, \beta_{1}, \beta_{2}$ and $\rho$ stationary state for the system $S$ interacting with both baths, By taking a sum over $j$ of the inequality before (3.2), we obtain

$$
\beta_{1} \operatorname{tr}\left(\rho \mathcal{L}_{1}\left(H_{S}\right)\right)+\beta_{2} \operatorname{tr}\left(\rho \mathcal{L}_{2}\left(H_{S}\right)\right) \leq 0
$$

Moreover, $\operatorname{tr}\left(\rho \mathcal{L}_{1}\left(H_{S}\right)\right)=-\operatorname{tr}\left(\rho \mathcal{L}_{2}\left(H_{S}\right)\right)$ and so

$$
\left(\beta_{2}-\beta_{1}\right) \operatorname{tr}\left(\rho \mathcal{L}_{2}\left(H_{S}\right)\right) \geq 0
$$

In view $\beta_{1} \geq \beta_{2}$, we have $\operatorname{tr}\left(\rho \mathcal{L}_{1}\left(H_{S}\right)\right)=-\operatorname{tr}\left(\rho \mathcal{L}_{2}\left(H_{S}\right)\right) \geq 0$ and the proof is complete.

In this section we prove a general explicit formula for the energy current in a stationary state $\rho$ which is a function of the system Hamiltonian $H_{S}$. This not only confirms that it is positive also for possibly infinite dimensional systems if the eigenvalues of stationary state are a monotone system (i.e. there are no population inversions), but it allows us to establish proportionality to the difference of bath temperatures when they are not too small, namely an approximate Fourier law.

Lemma 3.2. For all $\omega \in \mathrm{B}$ and $j=1,2$ we have

$$
\mathcal{Q}_{j, \omega}^{-}\left(H_{S}\right)=-\omega D_{j, \omega}^{*} D_{j, \omega} \quad \mathcal{Q}_{j, \omega}^{+}\left(H_{S}\right)=\omega D_{j, \omega} D_{j, \omega}^{*}
$$

and

$$
\mathcal{L}_{j}\left(H_{S}\right)=\sum_{\omega \in \mathrm{B}} \omega\left(\Gamma_{j, \omega}^{+} D_{j, \omega} D_{j, \omega}^{*}-\Gamma_{j, \omega}^{-} D_{j, \omega}^{*} D_{j, \omega}\right)
$$

Proof. Writing $H_{S}$ as in (2.1) we compute

$$
\begin{aligned}
\mathcal{Q}_{j, \omega}^{-}\left(H_{S}\right) & =-\frac{1}{2} D_{j, \omega}^{*} D_{j, \omega} H_{S}+D_{j, \omega}^{*} H_{S} D_{j, \omega}-\frac{1}{2} H_{S} D_{j, \omega}^{*} D_{j, \omega} \\
& =\sum_{\left(\varepsilon_{n}, \varepsilon_{m}\right) \in \mathrm{B}_{\omega}}\left(\varepsilon_{m} P_{\varepsilon_{n}} D_{j}^{*} P_{\varepsilon_{m}} D_{j} P_{\varepsilon_{n}}-\varepsilon_{n} P_{\varepsilon_{n}} D_{j}^{*} P_{\varepsilon_{m}} D_{j} P_{\varepsilon_{n}}\right) \\
& =-\sum_{\left(\varepsilon_{n}, \varepsilon_{m}\right) \in \mathrm{B}_{\omega}} \omega P_{\varepsilon_{n}} D_{j}^{*} P_{\varepsilon_{m}} D_{j} P_{\varepsilon_{n}} \\
& =-\omega D_{j, \omega}^{*} D_{j, \omega} .
\end{aligned}
$$

The proof of the other identity (3.3) is similar. Since $\left[H_{j, \omega}, H_{S}\right]=0$ for all $j, \omega,(3.4)$ follows immediately. 
We can now prove our formula for the energy current in a stationary state $\rho$ which is a function of the system Hamiltonian $H_{S}$. We suppose that the interaction of the system with both reservoirs is similar; this property is reflected by the assumptions on $\operatorname{tr}\left(P_{\varepsilon_{n}} D_{j}^{*} P_{\varepsilon_{m}} D_{j}\right)$ and $f_{1, \omega}$. In the sequel, to simplify the notation we also write $\rho_{n}$ instead of $\rho_{\varepsilon_{n}}$.

Theorem 3.3. For any state $\rho$ which is a function of the system Hamiltonian $H_{S}$, i.e.

$$
\rho=\sum_{n \geq 0} \rho_{n} P_{\varepsilon_{n}}
$$

we have

$$
\operatorname{tr}\left(\rho \mathcal{L}_{j}\left(H_{S}\right)\right)=\sum_{\omega \in \mathrm{B}} \omega \sum_{\left(\varepsilon_{n}, \varepsilon_{m}\right) \in \mathrm{B}_{\omega}}\left(\Gamma_{j, \omega}^{+} \rho_{m}-\Gamma_{j, \omega}^{-} \rho_{n}\right) \operatorname{tr}\left(P_{\varepsilon_{n}} D_{j}^{*} P_{\varepsilon_{m}} D_{j}\right) .
$$

If the state $\rho$ is also stationary and, moreover,

(1) $\operatorname{tr}\left(P_{\varepsilon_{n}} D_{1}^{*} P_{\varepsilon_{m}} D_{1}\right)=\operatorname{tr}\left(P_{\varepsilon_{n}} D_{2}^{*} P_{\varepsilon_{m}} D_{2}\right)$ for all $n, m$,

(2) $f_{1, \omega}=f_{2, \omega}$ for all $\omega$,

then

$$
\operatorname{tr}\left(\rho \mathcal{L}_{1}\left(H_{S}\right)\right)=\frac{1}{2} \sum_{\omega \in \mathbf{B}} \omega f_{1, \omega}\left(\gamma_{1, \omega}^{+}-\gamma_{2, \omega}^{+}\right) \sum_{\left(\varepsilon_{n}, \varepsilon_{m}\right) \in \mathrm{B}_{\omega}}\left(\rho_{m}-\rho_{n}\right) \operatorname{tr}\left(P_{\varepsilon_{n}} D_{1}^{*} P_{\varepsilon_{m}} D_{1}\right) .
$$

Proof. The proof of (3.6) is immediate from (3.4) and the following identities (cyclic property of the trace)

$$
\operatorname{tr}\left(P_{\varepsilon_{m}} D_{j, \omega} P_{\varepsilon_{n}} D_{j, \omega}^{*}\right)=\operatorname{tr}\left(\left(P_{\varepsilon_{m}} D_{j, \omega}\right) P_{\varepsilon_{m}} D_{j, \omega}^{*}\right)=\operatorname{tr}\left(P_{\varepsilon_{n}} D_{j, \omega}^{*} P_{\varepsilon_{m}} D_{j, \omega}\right) .
$$

If the state $\rho$ is stationary, then $\operatorname{tr}\left(\rho \mathcal{L}_{1}\left(H_{S}\right)\right)=\operatorname{tr}\left(\rho \mathcal{L}\left(H_{S}\right)\right)-\operatorname{tr}\left(\rho \mathcal{L}_{2}\left(H_{S}\right)\right)=-\operatorname{tr}\left(\rho \mathcal{L}_{2}\left(H_{S}\right)\right)$, so that $\operatorname{tr}\left(\rho \mathcal{L}_{1}\left(H_{S}\right)\right)=\left(\operatorname{tr}\left(\rho \mathcal{L}_{1}\left(H_{S}\right)\right)-\operatorname{tr}\left(\rho \mathcal{L}_{2}\left(H_{S}\right)\right)\right) / 2$. Computing the right-hand side difference by means of (3.6) with $j=1,2$ we can write $2 \operatorname{tr}\left(\rho \mathcal{L}_{1}\left(H_{S}\right)\right)$ as

$$
\begin{aligned}
& \sum_{\omega \in \mathrm{B}} \omega f_{1, \omega} \sum_{\left(\varepsilon_{n}, \varepsilon_{m}\right) \in \mathrm{B}_{\omega}}\left(\gamma_{1, \omega}^{+} \rho_{m}-\gamma_{1, \omega}^{-} \rho_{n}-\gamma_{2, \omega}^{+} \rho_{m}+\gamma_{2, \omega}^{-} \rho_{n}\right) \operatorname{tr}\left(P_{\varepsilon_{n}} D_{1}^{*} P_{\varepsilon_{m}} D_{1}\right) \\
= & \sum_{\omega \in \mathrm{B}} \omega f_{1, \omega} \sum_{\left(\varepsilon_{n}, \varepsilon_{m}\right) \in \mathrm{B}_{\omega}}\left(\left(\gamma_{1, \omega}^{+}-\gamma_{2, \omega}^{+}\right) \rho_{m}-\left(\gamma_{1, \omega}^{-}-\gamma_{2, \omega}^{-}\right) \rho_{n}\right) \operatorname{tr}\left(P_{\varepsilon_{n}} D_{1}^{*} P_{\varepsilon_{m}} D_{1}\right) .
\end{aligned}
$$

Since $\gamma_{j, \omega}^{-}=\gamma_{j, \omega}^{+}+1$ for all $j, \omega$, then $\gamma_{1, \omega}^{+}-\gamma_{2, \omega}^{+}=\gamma_{1, \omega}^{-}-\gamma_{2, \omega}^{-}$and (3.7) follows.

Remark. Note that the above identity $\operatorname{tr}\left(P_{\varepsilon_{n}} D_{1}^{*} P_{\varepsilon_{m}} D_{1}\right)=\operatorname{tr}\left(P_{\varepsilon_{n}} D_{2}^{*} P_{\varepsilon_{m}} D_{2}\right)$ holds whenever there exists an isometry $R$ on $\mathrm{h}$, commuting with $H_{S}$, such that $D_{2}=R D_{1} R^{*}$. Indeed, in this case, $R$ commutes with all spectral projections of $H_{S}$ and

$$
\begin{aligned}
\operatorname{tr}\left(P_{\varepsilon_{n}} D_{2}^{*} P_{\varepsilon_{m}} D_{2}\right) & =\operatorname{tr}\left(P_{\varepsilon_{n}} R D_{1}^{*} R^{*} P_{\varepsilon_{m}} R D_{1} R^{*}\right) \\
& =\operatorname{tr}\left(P_{\varepsilon_{n}} D_{1}^{*} P_{\varepsilon_{m}} D_{1} R^{*} R\right) \\
& =\operatorname{tr}\left(P_{\varepsilon_{n}} D_{1}^{*} P_{\varepsilon_{m}} D_{1}\right)
\end{aligned}
$$


We will see later (Section 5) that this happens when the system interacts in the same way with the two baths.

Formula (3.7) can be applied to effectively compute the energy current in several models highlighting the dependence on the difference of temperatures. Indeed, one readily sees that, for $\beta_{1}, \beta_{2}$ very close the term $\omega\left(\gamma_{1, \omega}^{+}-\gamma_{2, \omega}^{+}\right)$is an infinitesimum of order $\beta_{1}^{-1}-\beta_{2}^{-1}$ while the other terms are close to some nonzero values. Moreover, it is also clear from (3.7) that the energy current is non-negative whenever the invariant state satisfies $\rho_{m}>\rho_{n}$ for all $n, m$ such that $\varepsilon_{m}<\varepsilon_{n}$ i.e. population inversion does not occur.

However, in order to find more explicit formulae we need additional information on the invariant state. This problem will be studied in the next section. We end this section by the following example

Example 3.4. Let $\mathrm{h}=\mathbb{C}^{n+1}$ with orthonormal basis $\left(e_{k}\right)_{0 \leq k \leq n}$. Consider an $n$-level system with Hamiltonian

$$
H_{S}=\sum_{k=0}^{n} k\left|e_{k}\right\rangle\left\langle e_{k}\right|
$$

and interaction operators $D_{1}, D_{2}$ acting as

$$
D_{j} e_{k}=e_{k-1} \quad \text { for } k=1, \ldots, n \quad D_{j} e_{0}=0
$$

Clearly $\mathrm{B}=\{1,2, \ldots, n\}$ but the only nonzero $D_{j, \omega}$ are those corresponding to the frequency $\omega=1$ and $D_{1,1}=D_{1}, D_{2,1}=D_{2}$. Moreover, since $\varepsilon_{k}=k$,

$$
\operatorname{tr}\left(P_{\varepsilon_{k}} D_{1}^{*} P_{\varepsilon_{k-1}} D_{1}\right)=\operatorname{tr}\left(P_{\varepsilon_{k}} D_{2}^{*} P_{\varepsilon_{k-1}} D_{2}\right)=1
$$

for $k=1, \ldots, n$. By Theorem 3.3 formula (3.6) we have

$$
\operatorname{tr}\left(\rho \mathcal{L}_{j}\left(H_{S}\right)\right)=\sum_{k=0}^{n-1}\left(\Gamma_{j, 1}^{+} \rho_{k}-\Gamma_{j, 1}^{-} \rho_{k+1}\right) .
$$

If all $\Gamma_{j, 1}^{ \pm}(j=1,2)$ are nonzero, a straightforward computation shows that the unique stationary state is

$$
\rho=\frac{1-\nu}{1-\nu^{n+1}} \sum_{k=0}^{n} \nu^{k}\left|e_{k}\right\rangle\left\langle e_{k}\right|, \quad \nu:=\frac{\Gamma_{1,1}^{+}+\Gamma_{2,1}^{+}}{\Gamma_{1,1}^{-}+\Gamma_{2,1}^{-}}
$$

and the energy current due to interaction with reservoir $j$ is

$$
\begin{aligned}
\operatorname{tr}\left(\rho \mathcal{L}_{j}\left(H_{S}\right)\right) & =\frac{1-\nu}{1-\nu^{n+1}} \sum_{k=0}^{n-1}\left(\Gamma_{j, 1}^{+} \nu^{k}-\Gamma_{j, 1}^{-} \nu^{k+1}\right) \\
& =\frac{1-\nu^{n}}{1-\nu^{n+1}}\left(\Gamma_{j, 1}^{+}-\nu \Gamma_{j, 1}^{-}\right) .
\end{aligned}
$$

Note that, dropping the index 1 corresponding to the unique effective frequency $\omega$ to simplify the 
notation, we have

$$
\begin{aligned}
\Gamma_{j}^{+-}-\nu \Gamma_{j}^{-} & =\Gamma_{j}^{-}\left(\frac{\Gamma_{j}^{+}}{\Gamma_{j}^{-}}-\frac{\Gamma_{1}^{+}+\Gamma_{2}^{+}}{\Gamma_{1}^{-}+\Gamma_{2}^{-}}\right) \\
& =\Gamma_{j}^{-}\left(\frac{\gamma_{j}^{+}}{\gamma_{j}^{-}}-\frac{f_{1} \gamma_{1}^{+}+f_{2} \gamma_{2}^{+}}{f_{1} \gamma_{1}^{-}+f_{2} \gamma_{2}^{-}}\right) \\
& =\Gamma_{j}^{-}\left(\mathrm{e}^{-\beta_{j}}-\frac{f_{1}\left(\mathrm{e}^{\beta_{2}}-1\right)+f_{2}\left(\mathrm{e}^{\beta_{1}}-1\right)}{f_{1} \mathrm{e}^{\beta_{1}}\left(\mathrm{e}^{\beta_{2}}-1\right)+f_{2} \mathrm{e}^{\beta_{2}}\left(\mathrm{e}^{\beta_{1}}-1\right)}\right) \\
& =\Gamma_{j}^{-}\left(\mathrm{e}^{-\beta_{j}}-\frac{f_{1} \mathrm{e}^{-\beta_{1}}\left(1-\mathrm{e}^{-\beta_{2}}\right)+f_{2} \mathrm{e}^{-\beta_{2}}\left(1-\mathrm{e}^{-\beta_{1}}\right)}{f_{1}\left(1-\mathrm{e}^{-\beta_{2}}\right)+f_{2}\left(1-\mathrm{e}^{-\beta_{1}}\right)}\right) .
\end{aligned}
$$

For $j=1$ we find

$$
\Gamma_{j, 1}^{+}-\nu \Gamma_{j, 1}^{-}=\Gamma_{j}^{-} f_{2}\left(1-\mathrm{e}^{-\beta_{1}}\right) \frac{\mathrm{e}^{-\beta_{1}}-\mathrm{e}^{-\beta_{2}}}{f_{1}\left(1-\mathrm{e}^{-\beta_{2}}\right)+f_{2}\left(1-\mathrm{e}^{-\beta_{1}}\right)}
$$

and so

$$
\operatorname{tr}\left(\rho \mathcal{L}_{1}\left(H_{S}\right)\right)=\frac{1-\left(\left(\Gamma_{1}^{+}+\Gamma_{2}^{+}\right) /\left(\Gamma_{1}^{-}+\Gamma_{2}^{-}\right)\right)^{n}}{1-\left(\left(\Gamma_{1}^{+}+\Gamma_{2}^{+}\right) /\left(\Gamma_{1}^{-}+\Gamma_{2}^{-}\right)\right)^{n+1}} \frac{\Gamma_{1}^{-} f_{2}\left(1-\mathrm{e}^{-\beta_{1}}\right)\left(\mathrm{e}^{-\beta_{1}}-\mathrm{e}^{-\beta_{2}}\right)}{f_{1}\left(1-\mathrm{e}^{-\beta_{2}}\right)+f_{2}\left(1-\mathrm{e}^{-\beta_{1}}\right)}
$$

Since $\Gamma_{j}^{+}<\Gamma_{j}^{-}$, this formula, for $n$ big and $\beta_{1}, \beta_{2}$ small becomes

$$
\begin{aligned}
\operatorname{tr}\left(\rho \mathcal{L}_{1}\left(H_{S}\right)\right) & \approx \frac{f_{1} f_{2}\left(\mathrm{e}^{-\beta_{1}}-\mathrm{e}^{-\beta_{2}}\right)}{f_{1}\left(1-\mathrm{e}^{-\beta_{2}}\right)+f_{2}\left(1-\mathrm{e}^{-\beta_{1}}\right)} \\
& \approx \frac{f_{1} f_{2}\left(\beta_{2}-\beta_{1}\right)}{f_{2} \beta_{1}+f_{1} \beta_{2}} \\
& =\frac{f_{1} f_{2}\left(\frac{1}{\beta_{1}}-\frac{1}{\beta_{2}}\right)}{\frac{f_{1}}{\beta_{1}}+\frac{f_{2}}{\beta_{2}}}
\end{aligned}
$$

showing that, in a certain regime of high temperature a Fourier law holds for all choices $f_{1}, f_{2}$ of the interactions strength.

\section{Dependence of the energy current from temperature dif- ference and conductivity}

In this section we consider systems whose Hamiltonian $H_{S}$ has simple spectrum, namely each spectral projection $P_{\varepsilon_{n}}$ is one-dimensional, and make explicit the dependence of the energy current on the difference of temperatures $1 / \beta_{1}$ and $1 / \beta_{2}$.

We begin by noting that, if spectral projections $P_{\varepsilon_{n}}$ are one-dimensional one can associate with the open quantum system a classical (time continuous) Markov chain with state space $V$ the 
spectrum $\operatorname{sp}\left(H_{S}\right)$ of $H_{S}$ in a canonical way. Indeed, for every bounded function $f$ on $V$, we have

$$
\begin{aligned}
\mathcal{L}\left(f\left(H_{S}\right)\right) & =\sum_{n \geq 0} f\left(\varepsilon_{n}\right) \mathcal{L}\left(P_{\varepsilon_{n}}\right) \\
& =\sum_{\omega \in \mathrm{B},\left(\varepsilon_{n}, \varepsilon_{m}\right) \in \mathrm{B}_{\omega}}\left(\sum_{j} \Gamma_{j, \omega}^{-} P_{\varepsilon_{n}} D_{j}^{*} P_{\varepsilon_{m}} D_{j} P_{\varepsilon_{n}}\right)\left(f\left(\varepsilon_{m}\right)-f\left(\varepsilon_{n}\right)\right) \\
& +\sum_{\omega \in \mathrm{B},\left(\varepsilon_{n}, \varepsilon_{m}\right) \in \mathrm{B}_{\omega}}\left(\sum_{j} \Gamma_{j, \omega}^{+} P_{\varepsilon_{m}} D_{j} P_{\varepsilon_{n}} D_{j}^{*} P_{\varepsilon_{m}}\right)\left(f\left(\varepsilon_{n}\right)-f\left(\varepsilon_{m}\right)\right)
\end{aligned}
$$

and we find a classical Markov chain with transition rate matrix $Q=\left(q_{n m}\right)$

$$
q_{n m}= \begin{cases}\sum_{j} \Gamma_{j, \varepsilon_{n}-\varepsilon_{m}}^{-} \operatorname{tr}\left(D_{j}^{*} P_{\varepsilon_{m}} D_{j} P_{\varepsilon_{n}}\right), & \text { if } \varepsilon_{n}>\varepsilon_{m}, \\ \sum_{j} \Gamma_{j, \varepsilon_{m}-\varepsilon_{n}}^{+} \operatorname{tr}\left(D_{j} P_{\varepsilon_{m}} D_{j}^{*} P_{\varepsilon_{n}}\right), & \text { if } \varepsilon_{n}<\varepsilon_{m}, \\ -\sum_{m \neq n} q_{n m}, & \text { if } n=m .\end{cases}
$$

Now, if we consider the conditional expectation

$$
\mathcal{E}: \mathcal{B}(\mathrm{h}) \rightarrow \ell^{\infty}(V ; \mathbb{C}), \quad \mathcal{E}(x)=\sum_{m \geq 0} P_{\varepsilon_{m}} x P_{\varepsilon_{m}}
$$

where $\ell^{\infty}(V ; \mathbb{C})$ is the abelian algebra of bounded functions on $V$, we have that

$$
\mathcal{E} \circ \mathcal{L}=\mathcal{L} \circ \mathcal{E}
$$

Therefore, by defining the predual map $\mathcal{E}_{*}$ such that $\operatorname{tr}\left(\mathcal{E}_{*}(\rho) x\right)=\operatorname{tr}(\rho \mathcal{E}(x))$, if $\rho$ is an invariant state, we have also $0=\mathcal{E}_{*}\left(\mathcal{L}_{*}(\rho)\right)=\mathcal{L}_{*}\left(\mathcal{E}_{*}(\rho)\right)$ and

$$
\left(\pi_{n}\right) \mapsto \sum_{n \geq 0} \pi_{n} P_{\varepsilon_{n}}
$$

gives a one-to-one correspondence between diagonal invariant states of the open quantum system and invariant measures of the associated Markov chain.

In the following, in order to have at hand an explicit formula for the invariant measure, we suppose, for simplicity, that the graph associated with the above Markov chain is a path graph and jumps can occur only to nearest neighbour levels, namely $q_{n m}=0$ for $|n-m| \geq 2$. This assumption may hold, for instance, if the Hamiltonian $H_{S}$ is generic in the sense of [8], namely it is not only non-degenerate but also if $\varepsilon_{n}-\varepsilon_{m}=\varepsilon_{n^{\prime}}-\varepsilon_{m^{\prime}}$ then $\varepsilon_{n}=\varepsilon_{n^{\prime}}$ and $\varepsilon_{m}=\varepsilon_{m^{\prime}}$. Moreover, we assume that $q_{n m} \neq 0$ for $|n-m| \leq 1$. In this case the associated classical Markov chain has a simpler structure allowing one to make explicit computations and describe explicitly the structure of invariant states (see also [11] in a more general situation).

The explicit expression for the invariant state is $\rho=\sum_{n} \rho_{n} P_{\varepsilon_{n}}$ where

$$
\rho_{n}=\prod_{0 \leq k<n} \frac{q_{k, k+1}}{q_{k+1, k}} \rho_{0}
$$


with

$$
\begin{aligned}
& q_{k, k+1}=\sum_{j=1}^{2} \Gamma_{j, \varepsilon_{k+1}-\varepsilon_{k}}^{+} \operatorname{tr}\left(D_{j} P_{\varepsilon_{k+1}} D_{j}^{*} P_{\varepsilon_{k}}\right), \\
& q_{k+1, k}=\sum_{j=1}^{2} \Gamma_{j, \varepsilon_{k+1}-\varepsilon_{k}}^{-} \operatorname{tr}\left(D_{j}^{*} P_{\varepsilon_{k}} D_{j} P_{\varepsilon_{k+1}}\right)
\end{aligned}
$$

provided that the normalization condition

$$
\sum_{n \geq 1} \prod_{0 \leq k<n} \frac{q_{k, k+1}}{q_{k+1, k}}<+\infty
$$

holds, in which case $\rho_{0}$ is the inverse of the sum of the above series increased by 1 .

With the explicit formula for the invariant state we can find a Fourier's law for the energy current through the system. We begin by a technical lemma

Lemma 4.1. The following inequalities hold

$$
\mathrm{e}^{-\left(\beta_{1}+\beta_{2}\right) \omega / 2} \frac{\frac{1}{\beta_{1}}-\frac{1}{\beta_{2}}}{\frac{1}{\beta_{1}}+\frac{1}{\beta_{2}}} \leq \frac{\frac{1}{\mathrm{e}^{\beta_{1} \omega}-1}-\frac{1}{\mathrm{e}^{\beta_{2} \omega}-1}}{\frac{\mathrm{e}^{\beta_{1} \omega}}{\mathrm{e}^{\beta_{1} \omega}-1}+\frac{\mathrm{e}^{\beta_{2} \omega}}{\mathrm{e}^{\beta_{2} \omega}-1}} \leq \frac{\frac{1}{\beta_{1}}-\frac{1}{\beta_{2}}}{\frac{1}{\beta_{1}}+\frac{1}{\beta_{2}}},
$$

for all $0<\beta_{1} \leq \beta_{2}$ and $\omega>0$.

Proof. Note that $1 /\left(\mathrm{e}^{\beta_{1} \omega}-1\right)-1 /\left(\mathrm{e}^{\beta_{2} \omega}-1\right) \leq 1 /\left(\beta_{1} \omega\right)-1 /\left(\beta_{2} \omega\right)$ because the function $x \mapsto$ $1 /\left(\mathrm{e}^{x \omega}-1\right)-1 /(\omega x)$ is increasing on $] 0,+\infty[$ since

$$
\frac{\mathrm{d}}{\mathrm{d} x}\left(\frac{1}{\mathrm{e}^{x \omega}-1}-\frac{1}{\omega x}\right)=\frac{1}{\omega x^{2}}-\frac{\omega}{\left(\mathrm{e}^{\omega x / 2}-\mathrm{e}^{-\omega x / 2}\right)^{2}} \geq 0
$$

by the elementary inequality $\mathrm{e}^{\omega x / 2}-\mathrm{e}^{-\omega x / 2} \geq \omega x$. Moreover, by another elementary inequality $1-\mathrm{e}^{-\beta_{j} \omega} \leq \beta_{j} \omega$, we have

$$
\frac{\mathrm{e}^{\beta_{1} \omega}}{\mathrm{e}^{\beta_{1} \omega}-1}+\frac{\mathrm{e}^{\beta_{2} \omega}}{\mathrm{e}^{\beta_{2} \omega}-1}=\frac{1}{1-\mathrm{e}^{-\beta_{1} \omega}}+\frac{1}{1-\mathrm{e}^{-\beta_{2} \omega}} \geq \frac{1}{\beta_{1} \omega}+\frac{1}{\beta_{2} \omega}
$$

and the second inequality (4.4) follows.

In order to prove the first inequality we first write the right-hand side as

$$
\begin{aligned}
& \frac{\left(\mathrm{e}^{\beta_{1} \omega}-1\right)^{-1}-\left(\mathrm{e}^{\beta_{2} \omega}-1\right)^{-1}}{\mathrm{e}^{\beta_{1} \omega}\left(\mathrm{e}^{\beta_{1} \omega}-1\right)^{-1}+\mathrm{e}^{\beta_{2} \omega}\left(\mathrm{e}^{\beta_{2} \omega}-1\right)^{-1}} \\
= & \frac{\mathrm{e}^{\beta_{2} \omega}-\mathrm{e}^{\beta_{1} \omega}}{\mathrm{e}^{\beta_{1} \omega} \mathrm{e}^{\beta_{2} \omega / 2}\left(\mathrm{e}^{\beta_{2} \omega / 2}-\mathrm{e}^{-\beta_{2} \omega / 2}\right)+\mathrm{e}^{\beta_{2} \omega} \mathrm{e}^{\beta_{1} \omega / 2}\left(\mathrm{e}^{\beta_{1} \omega / 2}-\mathrm{e}^{-\beta_{1} \omega / 2}\right)} \\
= & \mathrm{e}^{-\left(\beta_{1}+\beta_{2}\right) \omega / 2} \frac{\mathrm{e}^{\left(\beta_{2}-\beta_{1}\right) \omega / 2}-\mathrm{e}^{-\left(\beta_{2}-\beta_{1}\right) \omega / 2}}{\left(1-\mathrm{e}^{-\beta_{2} \omega}\right)+\left(1-\mathrm{e}^{-\beta_{1} \omega}\right)}
\end{aligned}
$$


Noting that

$$
\begin{aligned}
\mathrm{e}^{\left(\beta_{2}-\beta_{1}\right) \omega / 2}-\mathrm{e}^{-\left(\beta_{2}-\beta_{1}\right) \omega / 2} & \geq 1+\frac{\left(\beta_{2}-\beta_{1}\right) \omega}{2}-\left(1-\frac{\left(\beta_{2}-\beta_{1}\right) \omega}{2}\right) \\
\left(1-\mathrm{e}^{-\beta_{2} \omega}\right)+\left(1-\mathrm{e}^{-\beta_{1} \omega}\right) & \leq\left(\beta_{1}+\beta_{2}\right) \omega
\end{aligned}
$$

we find

$$
\frac{\left(\mathrm{e}^{\beta_{1} \omega}-1\right)^{-1}-\left(\mathrm{e}^{\beta_{2} \omega}-1\right)^{-1}}{\mathrm{e}^{\beta_{1} \omega}\left(\mathrm{e}^{\beta_{1} \omega}-1\right)^{-1}+\mathrm{e}^{\beta_{2} \omega}\left(\mathrm{e}^{\beta_{2} \omega}-1\right)^{-1}} \geq \mathrm{e}^{-\left(\beta_{1}+\beta_{2}\right) \omega / 2} \frac{\left(\beta_{2}-\beta_{1}\right) \omega}{\left(\beta_{1}+\beta_{2}\right) \omega}
$$

This completes the proof.

Remark. Note that the inequalities of Lemma 4.1 provide a sharp estimate in terms of the inverse temperature difference $\beta_{2}-\beta_{1}$ for small $\beta_{1}, \beta_{2}$, i.e. when the average of temperatures $T_{1}, T_{2}$ is big. Indeed, the difference of the right-hand side and left-hand side is equal to

$$
\left(1-\mathrm{e}^{-\left(\beta_{1}+\beta_{2}\right) \omega / 2}\right) \frac{\beta_{2}-\beta_{1}}{\beta_{1}+\beta_{2}}
$$

and for temperatures $T_{j}>k_{B} \cdot 180 \mathrm{~K}=2.49 \cdot 10^{-21} \mathrm{~J}$ (approximately the lowest natural temperature ever recorded at ground level) we have $\beta_{j}<1 /\left(k_{B} \cdot 180 \mathrm{~K}\right)=4.02 \cdot 10^{20} \mathrm{~J}^{-1}$ so that the quantity that multiplies $\beta_{2}-\beta_{1}$ is

$$
\frac{1}{\beta_{1}+\beta_{2}}<1.24 \cdot 10^{-21} \mathrm{~J}
$$

Theorem 4.2. Suppose that

(1) $\operatorname{tr}\left(P_{\varepsilon_{n}} D_{j}^{*} P_{\varepsilon_{m}} D_{j}\right)=1$ for all $n, m$ and all $j=1,2$,

(2) $f_{j, \omega}=1$ for all $\omega$ and all $j=1,2$,

(3) Jumps can occur only to nearest neighbour levels,

(4) Formula (4.3) holds so that the state $\rho$ defined by (4.2) with $\rho_{0}$ determined by the normalization condition is invariant.

Then

$$
\kappa_{\mathrm{m}} \frac{\frac{1}{\beta_{1}}-\frac{1}{\beta_{2}}}{\frac{1}{\beta_{1}}+\frac{1}{\beta_{2}}} \kappa\left(\rho, H_{S}\right) \leq \operatorname{tr}\left(\rho \mathcal{L}_{1}\left(\mathcal{H}_{S}\right)\right) \leq \frac{\frac{1}{\beta_{1}}-\frac{1}{\beta_{2}}}{\frac{1}{\beta_{1}}+\frac{1}{\beta_{2}}} \kappa\left(\rho, H_{S}\right)
$$

where $\kappa_{\mathrm{m}}=\inf _{m \geq 0} \mathrm{e}^{-\left(\beta_{1}+\beta_{2}\right)\left(\varepsilon_{m+1}-\varepsilon_{m}\right) / 2}$ and

$$
\widehat{H}_{S}=\sum_{m \geq 0} \varepsilon_{m+1} P_{\varepsilon_{m}}, \quad \kappa\left(\rho, H_{S}\right)=\operatorname{tr}\left(\rho\left(\widehat{H}_{S}-H_{S}\right)\right) .
$$


Proof. By applying (3.7) in this context, we have

$$
\begin{aligned}
\operatorname{tr}\left(\rho \mathcal{L}_{1}\left(H_{S}\right)\right) & =\frac{1}{2} \sum_{n \geq 0}\left(\varepsilon_{n+1}-\varepsilon_{n}\right)\left(\rho_{n}-\rho_{n+1}\right)\left(\Gamma_{1, \varepsilon_{n+1}-\varepsilon_{n}}^{+}-\Gamma_{2, \varepsilon_{n+1}-\varepsilon_{n}}^{+}\right) \\
& =\frac{1}{2} \sum_{n \geq 0}\left(\varepsilon_{n+1}-\varepsilon_{n}\right)\left(1-\frac{q_{n, n+1}}{q_{n+1, n}}\right) \rho_{n}\left(\Gamma_{1, \varepsilon_{n+1}-\varepsilon_{n}}^{+}-\Gamma_{2, \varepsilon_{n+1}-\varepsilon_{n}}^{+}\right) \\
& =\sum_{n \geq 0}\left(\varepsilon_{n+1}-\varepsilon_{n}\right) \rho_{n} \frac{\Gamma_{1, \varepsilon_{n+1}-\varepsilon_{n}}^{+}-\Gamma_{2, \varepsilon_{n+1}-\varepsilon_{n}}^{+}}{\Gamma_{1, \varepsilon_{n+1}-\varepsilon_{n}}^{-}+\Gamma_{2, \varepsilon_{n+1}-\varepsilon_{n}}^{-}}
\end{aligned}
$$

Now the proof follows applying Lemma 4.1 with $\omega=\varepsilon_{n+1}-\varepsilon_{n}$ to estimate the right-hand side ratio.

Remark. Formula (4.5) shows that the energy current $\operatorname{tr}\left(\rho \mathcal{L}_{1}\left(H_{S}\right)\right)$ has an explicit dependence on the difference $\beta_{1}^{-1}-\beta_{2}^{-1}$ of the reservoirs' temperatures. This dependence holds only through two inequalities, but it suggests the existence of an "approximate" Fourier law (see $[4,21]$ ) for the current. Clearly there can be further dependecies through the term $\kappa\left(\rho, H_{S}\right)$, however it holds

$$
\inf _{k}\left(\varepsilon_{k+1}-\varepsilon_{k}\right) \leq \kappa\left(\rho, H_{S}\right) \leq \sup _{k}\left(\varepsilon_{k+1}-\varepsilon_{k}\right) .
$$

Therefore the energy current depends on the temperature difference mainly through the explicit term and one could say that there really is an "approximate" Fourier Law. Furthermore it is worth noticing that, for $\beta_{1}, \beta_{2}$ fixed, the inequality (4.5) is better the $\operatorname{smaller}$ is $\sup _{m \geq 0}\left(\varepsilon_{m+1}-\varepsilon_{m}\right)$ so that $\kappa_{\mathrm{m}}$ is close to 1 and the inequalities are approximately equalities. However, it should also be noted that, in this case, $\kappa\left(\rho, H_{S}\right)$ becomes small as well. Eventually note that, due to the nature of our system, we cannot investigate spatial properties of energy flow. Therefore our discussion of the Fourier's law is concerned with proportionality to temperature difference and not with dependency on size.

Remark. Since the above QMS are of weak coupling limit type, one can write explicitly the entropy production (in the sense of $[15,16]$ ).

It is tempting to study in detail what happens when $\sup _{m \geq 0}\left(\varepsilon_{m+1}-\varepsilon_{m}\right)$ tends to 0 so that the eigenvalues of $H_{S}$ increase in number and form a set more and more packed. In a more precise way, for all $n \geq 1$ we assume that the system Hamiltonian is a self-adjoint operator $H_{S}^{(n)}$ on an $(n+1)$-dimensional Hilbert space $\mathrm{h}$ with simple pure point spectrum $\left(\varepsilon_{k}^{(n)}\right)_{0 \leq k \leq n}$ with $\varepsilon_{0}=0$ and, for all $a, b$ with $0 \leq a<b \leq+\infty$, we have

$$
\left.\left.\lim _{n \rightarrow \infty} \frac{\operatorname{card}\left\{k \mid a<\varepsilon_{k}^{(n)} \leq b\right\}}{n}=\mu(] a, b\right]\right)
$$

for some continuous probability density $\mu$ on $[0,+\infty[$. In other words, the empirical distribution

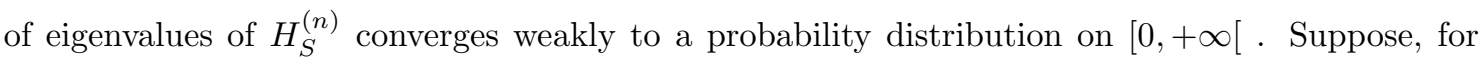
simplicity, that $\mu$ has no atoms, i.e. $\mu(\{r\})=0$ for all $r \geq 0$. 
We can now prove the following result on the distribution of eigenvalues of the stationary state and energy in stationary conditions.

Theorem 4.3. Under the assumptions of Theorem 4.2, for all $n \geq 1$, let $H_{S}^{(n)}$ be as above and suppose that (4.6) holds. Let $\rho^{(n)}$ be the invariant state (4.2) and let

$$
\widetilde{\beta}=2\left(1 / \beta_{1}+1 / \beta_{2}\right)^{-1}
$$

be the harmonic mean of the inverse temperatures (i.e. $\widetilde{\beta}^{-1}$ arithmetic mean of $\beta_{1}^{-1}, \beta_{2}^{-1}$ ).

(i) Eigenvectors $\rho_{k}^{(n)}$ of $\rho^{(n)}$ satisfy

$$
\lim _{n \rightarrow \infty} \sum_{\left\{k \mid a<\varepsilon_{k} \leq b\right\}} \rho_{k}^{(n)}=\frac{\int_{a}^{b} \mathrm{e}^{-\widetilde{\beta} r} \mathrm{~d} \mu(r)}{\int_{0}^{\infty} \mathrm{e}^{-\widetilde{\beta} r} \mathrm{~d} \mu(r)}
$$

(ii) The average energy in the system satisfies

$$
\lim _{n \rightarrow \infty} \operatorname{tr}\left(\rho^{(n)} H_{S}^{(n)}\right)=\frac{\int_{0}^{\infty} \mathrm{e}^{-\widetilde{\beta} r} r \mathrm{~d} \mu(r)}{\int_{0}^{\infty} \mathrm{e}^{-\widetilde{\beta} r} \mathrm{~d} \mu(r)} .
$$

This result reminds the one in [20] where the steady state can be described by a generalized Gibbs state and the steady-state current is proportional to the difference in the reservoirs' magnetizations.

In the proof we need the following Lemma.

Lemma 4.4. Let $\widetilde{\beta}=2 /\left(\beta_{1}^{-1}+\beta_{2}^{-1}\right)$ be the harmonic mean of inverse temperatures (i.e. $\widetilde{\beta}^{-1}$ is the arithmetic mean of $\beta_{1}^{-1}$ and $\left.\beta_{2}^{-1}\right)$. For all $1 \leq k \leq n$ and for $\sup _{j} \omega_{j}<1 /\left(3 \beta_{2}\right)$,

$$
1-\widetilde{\beta} \omega_{k} \leq \frac{q_{k, k+1}}{q_{k+1, k}} \leq 1-\widetilde{\beta} \omega_{k}+\left(\widetilde{\beta} \omega_{k}\right)^{2}
$$

where $\omega_{k}=\varepsilon_{k+1}-\varepsilon_{k}$ and

$$
\mathrm{e}^{-\widetilde{\beta} \varepsilon_{k}\left(1+\widetilde{\beta} \sup _{j} \omega_{j}\right)} \leq \prod_{j=0}^{k-1} \frac{q_{j, j+1}}{q_{j+1, j}} \leq \mathrm{e}^{-\widetilde{\beta} \varepsilon_{k}\left(1-\widetilde{\beta} \sup _{j} \omega_{j}\right)}
$$

Proof. By the elementary inequality $1-\mathrm{e}^{-\beta_{j} \omega_{k}} \leq \beta_{j} \omega_{k}$ we have

$$
\begin{aligned}
\frac{q_{k, k+1}}{q_{k+1, k}} & =\frac{\frac{1}{\mathrm{e}^{\beta_{1} \omega_{k}}-1}+\frac{1}{\mathrm{e}^{\beta_{2} \omega_{k}}-1}}{\frac{\mathrm{e}^{\beta_{1} \omega_{k}}}{\mathrm{e}^{\beta_{1} \omega_{k}}-1}+\frac{\mathrm{e}^{\beta_{2} \omega_{k}}}{\mathrm{e}^{\beta_{2} \omega_{k}}-1}} \\
& =1-\frac{2}{\left(1-\mathrm{e}^{-\beta_{1} \omega_{k}}\right)^{-1}+\left(1-\mathrm{e}^{-\beta_{2} \omega_{k}}\right)^{-1}} \\
& \geq 1-\frac{2 \omega_{k}}{1 / \beta_{1}+1 / \beta_{2}}
\end{aligned}
$$


In the same way, by the elementary inequalities $1-\mathrm{e}^{-\beta_{j} \omega_{k}} \geq \beta_{j} \omega_{k}-\left(\beta_{j} \omega_{k}\right)^{2} / 2$ and $1 /\left(1-\beta_{j} \omega_{k} / 2\right) \leq$ $1+\beta_{j} \omega_{k}$, we find for $\beta_{j} \omega_{k}<1$

$$
\begin{aligned}
\frac{q_{k, k+1}}{q_{k+1, k}} & \leq 1-\frac{2 \omega_{k}}{1 /\left(\beta_{1}\left(1-\beta_{1} \omega_{k} / 2\right)\right)+1 /\left(\beta_{2}\left(1-\beta_{2} \omega_{k} / 2\right)\right)} \\
& \leq 1-\frac{2 \omega_{k}}{1 / \beta_{1}\left(1+\beta_{1} \omega_{k} / 2\right)+1 / \beta_{2}\left(1+\beta_{2} \omega_{k} / 2\right)} \\
& \leq 1-\frac{2 \omega_{k}}{1 / \beta_{1}+1 / \beta_{2}+2 \omega_{k}} \\
& =1-\frac{\widetilde{\beta} \omega_{k}}{1+\widetilde{\beta} \omega_{k}}
\end{aligned}
$$

and so (4.7) follows.

In order to prove the upper bound in $(4.8)$, note that, since $\log (1-x) \leq-x$

$$
\log \left(\prod_{j=0}^{k-1} \frac{q_{j, j+1}}{q_{j+1, j}}\right) \leq \sum_{j=0}^{k-1} \log \left(1-\widetilde{\beta} \omega_{j}\left(1-\widetilde{\beta} \omega_{j}\right)\right) \leq-\sum_{j=0}^{k-1} \widetilde{\beta} \omega_{j}\left(1-\widetilde{\beta} \omega_{j}\right),
$$

as a consequence

$$
\log \left(\prod_{j=0}^{k-1} \frac{q_{j, j+1}}{q_{j+1, j}}\right) \leq-\sum_{j=0}^{k-1} \widetilde{\beta} \omega_{j}\left(1-\widetilde{\beta} \sup _{l} \omega_{l}\right)=-\widetilde{\beta} \varepsilon_{k}\left(1-\widetilde{\beta} \sup _{l} \omega_{l}\right) .
$$

For the lower bound, we begin by the inequality

$$
\log \left(\prod_{j=0}^{k-1} \frac{q_{j, j+1}}{q_{j+1, j}}\right)=\sum_{j=0}^{k-1} \log \left(\frac{q_{j, j+1}}{q_{j+1, j}}\right) \geq \sum_{j=0}^{k-1} \log \left(1-\widetilde{\beta} \omega_{j}\right) .
$$

Note that $\log (1-x)+x+x^{2} \geq 0$ for $0 \leq x \leq 2 / 3$ and, since $\widetilde{\beta} \omega_{j}<2 / 3$ by our assumption, we have

$$
\log \left(\prod_{j=0}^{k-1} \frac{q_{j, j+1}}{q_{j+1, j}}\right) \geq-\sum_{j=0}^{k-1} \widetilde{\beta} \omega_{j}\left(1+\widetilde{\beta} \sup _{l} \omega_{l}\right)=-\widetilde{\beta} \epsilon_{k}\left(1+\widetilde{\beta} \sup _{l} \omega_{l}\right) .
$$

This completes the proof.

Proof of Theorem 4.3. Let $\mu_{n}$ be the empirical distribution of the eigenvalues of $H_{S}^{(n)}$ i.e.

$$
\mu_{n}=\frac{1}{n+1} \sum_{k=0}^{n} \delta_{\varepsilon_{k}}
$$

and note that

$$
\sum_{\left\{k \mid a<\varepsilon_{k} \leq b\right\}} \rho_{k}^{(n)}=\frac{\frac{1}{n+1} \sum_{\left\{k \mid a<\varepsilon_{k} \leq b\right\}} \prod_{j=0}^{k-1} \frac{q_{j, j+1}}{q_{j+1, j}}}{\frac{1}{n+1} \sum_{k=0}^{n} \prod_{j=0}^{k-1} \frac{q_{j, j+1}}{q_{j+1, j}}} .
$$


Clearly, by Lemma 4.4,

$$
\begin{aligned}
\frac{1}{n+1} \sum_{\left\{k \mid a<\varepsilon_{k} \leq b\right\}} \prod_{j=0}^{k-1} \frac{q_{j, j+1}}{q_{j+1, j}} & \leq \frac{1}{n+1} \sum_{\left\{k \mid a<\varepsilon_{k} \leq b\right\}} \mathrm{e}^{-\tilde{\beta} \varepsilon_{k}\left(1-\widetilde{\beta} \sup _{j} \omega_{j}\right)} \\
& \leq \mathrm{e}^{\widetilde{\beta}^{2} b \sup _{j} \omega_{j}} \int_{] a, b]} \mathrm{e}^{-\tilde{\beta} \varepsilon_{k}} \mathrm{~d} \mu_{n}(r)
\end{aligned}
$$

and also

$$
\begin{aligned}
\frac{1}{n+1} \sum_{\left\{k \mid a<\varepsilon_{k} \leq b\right\}} \prod_{j=0}^{k-1} \frac{q_{j, j+1}}{q_{j+1, j}} & \geq \frac{\mathrm{e}^{-\widetilde{\beta}^{2} a \sup _{j} \omega_{j}}}{n+1} \sum_{\left\{k \mid a<\varepsilon_{k} \leq b\right\}} \mathrm{e}^{-\widetilde{\beta} \varepsilon_{k}} \\
& =\mathrm{e}^{-\widetilde{\beta}^{2} a \sup _{j} \omega_{j}} \int_{] a, b]} \mathrm{e}^{-\tilde{\beta} \varepsilon_{k}} \mathrm{~d} \mu_{n}(r) .
\end{aligned}
$$

Since $\sup _{j} \omega_{j}$ goes to 0 , probability measures $\mu_{n}$ converge weakly to $\mu$ and the function $r \rightarrow \mathrm{e}^{-\widetilde{\beta} r}$ is bounded continuous on $[0,+\infty[$, taking the limit as $n \rightarrow \infty$, we have

$$
\lim _{n \rightarrow \infty} \frac{1}{n+1} \sum_{\left\{k \mid a<\varepsilon_{k} \leq b\right\}} \prod_{j=0}^{k-1} \frac{q_{j, j+1}}{q_{j+1, j}}=\int_{] a, b]} \mathrm{e}^{-\tilde{\beta} \varepsilon_{k}} \mathrm{~d} \mu(r) .
$$

In the same way, taking $a=0$ and $b=+\infty$, we see that the denominator of (4.9) converges to

$$
\int_{0}^{+\infty} \mathrm{e}^{-\tilde{\beta} r} \mathrm{~d} \mu(r)
$$

and the proof of (i) is complete. The proof of (ii) is similar.

Remark. Theorem 4.3 (i) shows that, if $\mu$ has density $\mu^{\prime}$, then the asymptotic distribution of eigenvalues of the stationary state is

$$
\lambda \mapsto \frac{\mathrm{e}^{-\tilde{\beta} \lambda} \mu^{\prime}(\lambda)}{\int_{0}^{+\infty} \mathrm{e}^{-\tilde{\beta} r} \mu^{\prime}(r) \mathrm{d} r} .
$$

The asymptotic average energy in the system can be easily computed in some remarkable cases noting that the integral of $\mathrm{e}^{-\tilde{\beta} r}$ with respect to $\mu$ is the moment generating function $\phi$ of $\mu$ evaluated at $-\tilde{\beta}$ and so the asymptotic average energy in the system is

$$
-\frac{\frac{\mathrm{d}}{\mathrm{d} \tilde{\beta}} \phi(-\tilde{\beta})}{\phi(-\tilde{\beta})}=-\frac{\mathrm{d}}{\mathrm{d} \tilde{\beta}} \log (\phi(-\tilde{\beta})) .
$$

We can easily find an explicit result in two cases:

$$
\begin{array}{rll}
\mu \text { normal distribution } N\left(m, \sigma^{2}\right) & \text { average energy } & m-\tilde{\beta} \sigma \\
\mu \text { gamma distribution } \Gamma(\alpha, \theta) & \text { average energy } & \alpha /(\tilde{\beta}+\theta)
\end{array}
$$

The asymptotic average energy in the system is decreasing in $\tilde{\beta}$, i.e. increasing in the average temperature as expected, for all probability measure $\mu$ because the moment generating function of a probability distribution is log-convex and the derivative of a convex function is increasing.

Remark. Note that, by choosing a suitable spacing of eigenvalues $\varepsilon_{n}$ we can control the rate of convergence to 0 of $\kappa\left(\rho^{(n)}, H_{S}^{(n)}\right)$ at will, as $n$ tends to $+\infty$. 


\section{One dimensional Ising chain}

In this section we consider a one-dimensional Ising chain with nearest neighbour interaction. We will show that, in this case, if the heat baths interact locally at both ends of the chain, then the energy current is zero. Spin interaction (see 5.1) occurs only in the $z$ component. In the case where also the other components interact the derivation of the GKSL generator turns out to be really difficult (see [5]). Indeed, starting from the diagonalized $H_{S}$, one finds a cumbersome expression for the operators $D_{\omega}$.

In spite of the simple system Hamitonian $H_{S}$ (5.1) Theorems 4.2 and 4.3 do not apply to this model because its spectrum is degenerate.

The system space is $\mathrm{h}=\mathbb{C}^{2 \otimes N}$ with $N>2$. Define Pauli matrices

$$
\sigma^{x}=\left[\begin{array}{cc}
0 & 1 \\
1 & 0
\end{array}\right] \quad \sigma^{y}=\left[\begin{array}{cc}
0 & -\mathrm{i} \\
\mathrm{i} & 0
\end{array}\right] \quad \sigma^{z}=\left[\begin{array}{cc}
1 & 0 \\
0 & -1
\end{array}\right]
$$

with respect to the orthonormal basis $e_{+}=[1,0]^{\mathrm{T}}, e_{-}=[0,1]^{\mathrm{T}}$ of $\mathbb{C}^{2}$.

Consider the one dimensional Ising chain with Hamiltonian

$$
H_{S}=J_{z} \sum_{j=1}^{N-1} \sigma_{j}^{z} \sigma_{j+1}^{z}, \quad J_{z}>0, N>2
$$

Subsequently let us define

$$
e_{\alpha}:=\otimes_{j=1}^{N} e_{\alpha(j)}, \quad \alpha \in\{-1,1\}^{N},
$$

as a basis of $\mathrm{h}$, where $e_{-1}:=e_{-}$and $e_{+1}:=e_{+}$. Vectors $\left\{e_{\alpha}\right\}_{\alpha}$ form an eigenbasis for $H_{S}$ and the spectrum is

$$
\operatorname{sp}\left(H_{S}\right)=\left\{J_{z}(2 k-(N-1)) \mid k=0, \ldots, N-1\right\} .
$$

The eigenspace associated with the eigenvalue $\varepsilon_{k}=J_{z}(2 k-(N-1))$ is the linear span of the elements $e_{\alpha}$ such that exactly $k$ neighbouring elements in $\alpha$ have the same sign. Thus one can define the sets

$$
A_{k}:=\left\{\alpha \in\{-1,1\}^{N} \mid \sum_{j=1}^{N-1} \alpha(j) \alpha(j+1)=2 k-(N-1)\right\},
$$

and the spectral projection associated with the eigenvalue $\varepsilon_{k}$ is given by

$$
P_{k}:=\sum_{\alpha \in A_{k}}\left|e_{\alpha}\right\rangle\left\langle e_{\alpha}\right|
$$

The system is coupled with two heat reservoirs at inverse temperature $\beta_{1}, \beta_{2}$ with $\beta_{1} \leq \beta_{2}$ through the interaction

$$
H_{1}=\sigma_{1}^{u} \otimes\left(A^{-}\left(\phi_{1}\right)+A^{+}\left(\phi_{1}\right)\right), \quad H_{2}=\sigma_{N}^{v} \otimes\left(A^{-}\left(\phi_{2}\right)+A^{+}\left(\phi_{2}\right)\right),
$$


where $u, v \in \mathbb{R}^{3}$ and $\sigma_{i}^{u}$ is defined as

$$
\sigma_{i}^{u}=u_{1} \sigma_{i}^{x}+u_{2} \sigma_{i}^{y}+u_{3} \sigma_{i}^{z}
$$

The set of positive Bohr frequencies is given by

$$
\mathrm{B}:=\left\{2 J_{z}(n-m)=\varepsilon_{n}-\varepsilon_{m} \mid n, m \in\{0, \ldots, N-1\}, n>m\right\},
$$

while the operators $D_{j, \omega}$ are given by (2.5). Thus one has

$$
D_{1,2 J_{z}}=\left(u_{1}-\mathrm{i} u_{2}\right) \sum_{\alpha \in C_{++}^{l}} \sigma_{1}^{x}\left|e_{\alpha}\right\rangle\left\langle e_{\alpha}\left|+\left(u_{1}+\mathrm{i} u_{2}\right) \sum_{\alpha \in C_{--}^{l}} \sigma_{1}^{x}\right| e_{\alpha}\right\rangle\left\langle e_{\alpha}\right|
$$

where $C_{++}^{l}$ (resp. $C_{--}^{l}$ ) denotes the set of configurations $\alpha \in\{-1,+1\}^{N}$ with ++ (resp. --) in the first two sites ( $l$ stands for left). While $D_{1, \omega}=0$ for every $\omega \in \mathrm{B}-\left\{2 J_{z}\right\}$ because the Pauli matrices act only on the first site and so the number of neighbouring sites with the same sign can vary of at most one after the action of $\sigma_{1}^{u}$ and for $\omega=2 J_{z}$ one has

$$
D_{1,2 J_{z}}=\sum_{n=1}^{N-1} \sum_{\alpha \in A_{n}} \sum_{\beta \in A_{n+1}}\left\langle e_{\alpha}, \sigma_{1}^{x} e_{\beta}\right\rangle\left|e_{\alpha}\right\rangle\left\langle e_{\beta}\right| .
$$

With similar arguments one can see that $D_{2, \omega}=0$ for every $\omega \in \mathrm{B}-\left\{2 J_{z}\right\}$, while

$$
D_{2,2 J_{z}}=\left(v_{1}-\mathrm{i} v_{2}\right) \sum_{\alpha \in C_{++}^{r}} \sigma_{N}^{x}\left|e_{\alpha}\right\rangle\left\langle e_{\alpha}\left|+\left(v_{1}+\mathrm{i} v_{2}\right) \sum_{\alpha \in C_{--}^{r}} \sigma_{N}^{x}\right| e_{\alpha}\right\rangle\left\langle e_{\alpha}\right|
$$

where $C_{++}^{r}$ (resp. $C_{--}^{r}$ ) denotes the set of configurations with ++ (resp. --) in the last two sites ( $r$ stands for right).

From now on we will drop the subscript $2 J_{z}$ and only deal with operators related to that Bohr frequency, as the others vanish.

Recalling the definition of linear maps (2.6) and the constants

$$
\gamma_{i}^{+}=1 /\left(\mathrm{e}^{2 J_{z} \beta_{i}}-1\right), \quad \gamma_{i}^{-}=\mathrm{e}^{2 J_{z} \beta_{i}} /\left(\mathrm{e}^{2 J_{z} \beta_{i}}-1\right),
$$

we can write the GKSL generator of the evolution as follows

$$
\mathcal{L}=\sum_{i \in\{1, N\}} \gamma_{i}^{-} \mathcal{Q}_{i}^{-}+\gamma_{i}^{+} \mathcal{Q}_{i}^{+}
$$

A close scrutiny at the operators $D_{i}, D_{i}^{*}$ shows that, for each fixed configuration $\bar{\alpha} \in\{-1,+1\}^{N-2}$ of the $N-2$ inner sites of the chain the 4 -dimensional projections $p_{\bar{\alpha}}$ on subspaces

$$
\mathrm{h}_{\bar{\alpha}}:=\operatorname{span}\left\{e_{\alpha} \mid \alpha(j)=\bar{\alpha}(j) \text { for all } 2 \leq j \leq N-1 ; \alpha(1), \alpha(N) \in\{-1,1\}\right\}
$$

commute with both $D_{i}$ and $D_{i}^{*}$ for $i \in\{1, N\}$, then subalgebras $p_{\alpha_{1}} \mathcal{B}(\mathrm{h}) p_{\alpha_{2}}$ are invariant for the semigroup $\mathcal{T}$ generated by $\mathcal{L}$. This commutation allows us to restrict our study only to cases where 
the invariant state is of the form

$$
\rho=\sum_{\bar{\alpha} \in\{-1,1\}^{N-2}} p_{\bar{\alpha}} \rho p_{\bar{\alpha}}=\sum_{\bar{\alpha} \in\{-1,1\}^{N-2}} \lambda_{\bar{\alpha}} \rho_{\bar{\alpha}},
$$

where $\rho_{\bar{\alpha}}$ is an invariant state supported only on $h_{\bar{\alpha}}$ and $\lambda_{\bar{\alpha}}$ are real constants that sum up to 1 . Indeed the off diagonal terms, $p_{\overline{\alpha_{1}}} \rho p_{\overline{\alpha_{2}}}$ with $\alpha_{1} \neq \alpha_{2}$, do not contribute to current flow, since

$$
\operatorname{tr}\left(p_{\overline{\alpha_{1}}} \rho p_{\overline{\alpha_{2}}} \mathcal{L}_{1}\left(H_{S}\right)\right)=\operatorname{tr}\left(p_{\overline{\alpha_{1}}} \rho \mathcal{L}_{1}\left(H_{S}\right) p_{\overline{\alpha_{2}}}\right)=0
$$

Moreover all the conditional expectations $\mathcal{E}_{\bar{\alpha}}(x):=p_{\bar{\alpha}} x p_{\bar{\alpha}}$ commute with $\mathcal{L}$, ensuring that both $\sum_{\bar{\alpha}} \mathcal{E}_{\bar{\alpha}, *}(\rho)$ and every $\mathcal{E}_{\bar{\alpha}, *}(\rho)$ must also be invariant states on their own. As a further refinement we can repeat the same argument using the conditional expectation $\mathcal{E}(x):=\sum_{k=0}^{N-1} P_{k} x P_{k}$. Indeed $\mathcal{E}$ commutes with the Lindbladian $\mathcal{L}$ and

$$
\operatorname{tr}\left(P_{k_{1}} \rho P_{k_{2}} \mathcal{L}_{1}\left(H_{S}\right)\right)=\operatorname{tr}\left(P_{k_{1}} \rho \mathcal{L}_{1}\left(H_{S}\right) P_{k_{2}}\right)=0
$$

for $k_{1} \neq k_{2}$, since the spectral projections commute with $D_{j} D_{j}^{*}, D_{j}^{*} D_{j}$ and $\mathcal{L}_{1}\left(H_{S}\right)$ is a linear combination of these operators by Lemma 3.2, equation (3.3). In this way we can focus our study on invariant states of the form (5.3) with

$$
p_{\bar{\alpha}} \rho p_{\bar{\alpha}}=\rho_{\bar{\alpha}}=\left[\begin{array}{cccc}
\rho_{11}^{\bar{\alpha}} & 0 & 0 & 0 \\
0 & \rho_{22}^{\bar{\alpha}} & \rho_{23}^{\bar{\alpha}} & 0 \\
0 & \rho_{32}^{\bar{\alpha}} & \rho_{33}^{\bar{\alpha}} & 0 \\
0 & 0 & 0 & \rho_{44}^{\bar{\alpha}}
\end{array}\right]
$$

where we expanded the state with respect to the basis of four vectors $e_{c} \bar{\alpha} c, e_{d \bar{\alpha} c}, e_{c \bar{\alpha} d}, e_{d \bar{\alpha} d}$ defined as follows: $e_{c \bar{\alpha} c}$ is the vector $e_{\bar{\alpha}(2), \bar{\alpha}(2), \ldots, \bar{\alpha}(N-1), \bar{\alpha}(N-1)}, e_{c \bar{\alpha} d}=e_{\bar{\alpha}(2), \bar{\alpha}(2), \ldots, \bar{\alpha}(N-1),-\bar{\alpha}(N-1)}$ and vectors $e_{d \bar{\alpha} c}, e_{d \bar{\alpha} d}$ are defined in a similar way.

Now we have reduced and simplified the class of states we want to use when looking for a invariant state, without, however, losing any contribution to the current flow. In order to find the invariant state, first of all it is not too difficult to show that $\mathcal{L}_{*}$ leaves invariant the subspace of diagonal elements. Then compute

$$
\mathcal{L}_{*}\left(\rho_{23}^{\bar{\alpha}}\left|e_{d \alpha c}\right\rangle\left\langle e_{c \alpha d}\right|\right)=-\frac{1}{2}\left[\Gamma_{1}^{+}+\Gamma_{1}^{-}+\Gamma_{N}^{+}+\Gamma_{N}^{-}\right] \rho_{23}^{\bar{\alpha}}\left|e_{d \alpha c}\right\rangle\left\langle e_{c \alpha d}\right|,
$$

and similarly

$$
\mathcal{L}_{*}\left(\rho_{32}^{\bar{\alpha}}\left|e_{c \alpha d}\right\rangle\left\langle e_{d \alpha c}\right|\right)=-\frac{1}{2}\left[\Gamma_{1}^{+}+\Gamma_{1}^{-}+\Gamma_{N}^{+}+\Gamma_{N}^{-}\right] \rho_{32}^{\bar{\alpha}}\left|e_{c \alpha d}\right\rangle\left\langle e_{d \alpha c}\right|
$$

where $\Gamma_{1}^{ \pm}=\left\|u_{1}+\mathrm{i} u_{2}\right\|^{2} \gamma_{1}^{ \pm}$and $\Gamma_{N}^{ \pm}=\left\|v_{1}+\mathrm{i} v_{2}\right\|^{2} \gamma_{N}^{ \pm}$. (The above $\Gamma_{i}^{ \pm}$slightly differ from the constants in Section 2). Therefore the invariant state condition $\mathcal{L}_{*}(\rho)=0$ implies $\rho_{23}^{\bar{\alpha}}=\rho_{32}^{\bar{\alpha}}=0$. 
We can now just consider the reduced dynamics on diagonal elements of $p_{\bar{\alpha}} \mathcal{B}(\mathrm{h}) p_{\bar{\alpha}}$, given by

$$
\mathcal{L}_{*}=\left[\begin{array}{cccc}
-\left(\Gamma_{1}^{-}+\Gamma_{N}^{-}\right) & \Gamma_{1}^{-} & \Gamma_{N}^{-} & 0 \\
\Gamma_{1}^{+} & -\left(\Gamma_{1}^{+}+\Gamma_{N}^{-}\right) & 0 & \Gamma_{N}^{-} \\
\Gamma_{N}^{+} & 0 & -\left(\Gamma_{N}^{+}+\Gamma_{1}^{-}\right) & \Gamma_{1}^{-} \\
0 & \Gamma_{N}^{+} & \Gamma_{1}^{+} & -\left(\Gamma_{N}^{+}+\Gamma_{1}^{+}\right)
\end{array}\right],
$$

The unique invariant law for the time-continuous Markov chain generated by the above matrix is

$$
\pi=Z^{-1}\left[1, \mathrm{e}^{2 J_{z} \beta_{1}}, \mathrm{e}^{2 J_{z} \beta_{2}}, \mathrm{e}^{2 J_{z}\left(\beta_{1}+\beta_{2}\right)}\right],
$$

where $Z^{-1}$ is a normalization constant that is independent of $u, v$ and is the same for all $\bar{\alpha}$. Therefore the unique $\mathcal{T}$-invariant state supported on $\mathrm{h}_{\alpha}$ is

$$
\begin{aligned}
\rho_{\alpha} & =Z^{-1}\left(\left|e_{c \bar{\alpha} c}\right\rangle\left\langle e_{c \bar{\alpha} c}\left|+\mathrm{e}^{2 J_{z} \beta_{1}}\right| e_{d \bar{\alpha} c}\right\rangle\left\langle e_{d \bar{\alpha} c}\right|\right. \\
& \left.+\mathrm{e}^{2 J_{z} \beta_{2}}\left|e_{c \bar{\alpha} d}\right\rangle\left\langle e_{c \bar{\alpha} d}\left|+\mathrm{e}^{2 J_{z}\left(\beta_{1}+\beta_{2}\right)}\right| e_{d \bar{\alpha} d}\right\rangle\left\langle e_{d \bar{\alpha} d}\right|\right) .
\end{aligned}
$$

Recalling (5.3) we can now write any invariant state for the semigroup $\mathcal{T}$.

We can now evaluate the energy flow $\operatorname{tr}\left(\rho \mathcal{L}_{1}\left(H_{S}\right)\right)$ via the expression

$$
\mathcal{L}_{1}\left(H_{S}\right)=\sum_{\omega \in B^{+}} \omega\left(\gamma_{1, \omega}^{+} D_{1} D_{1}^{*}-\gamma_{1, \omega}^{-} D_{1}^{*} D_{1}\right)=2 J_{z}\left(\gamma_{1}^{+} D_{1} D_{1}^{*}-\gamma_{1}^{-} D_{1}^{*} D_{1}\right)
$$

that, together with the formula for $\rho_{\alpha}$, yields

$$
\begin{aligned}
Z \operatorname{tr}\left(\rho \mathcal{L}_{1}\left(H_{S}\right)\right) & =Z \operatorname{tr}\left(\sum_{\bar{\alpha} \in\{-1,1\}^{N-2}} \lambda_{\bar{\alpha}} \rho_{\bar{\alpha}} \mathcal{L}_{1}\left(H_{S}\right)\right) \\
& =\sum_{\bar{\alpha} \in\{-1,1\}^{N-2}} 2 J_{z} \lambda_{\bar{\alpha}}\left(\gamma_{1}^{+} \mathrm{e}^{\beta_{1} \omega}+\gamma_{1}^{+} \mathrm{e}^{\left(\beta_{1}+\beta_{2}\right) \omega}-\gamma_{1}^{-} \mathrm{e}^{\beta_{2} \omega}-\gamma_{1}^{-}\right) \\
& =0
\end{aligned}
$$

Remark. For $N=2$, it can be shown by direct computation that the energy current is strictly positive. Indeed, because of low dimensionality the ends of the chain can interact directly.

\section{Acknowledgement.}

The authors would like to thank Stefano Olla for drawing their attention to the problem of energy transport in quantum systems and fruitful discussions at the workshop "Quantum Transport Equations and Applications" at Casa Matemática Oaxaca (México) in September 2018. They also gratefully thank CUBO's anonymous referees whose remarks and comments to improve an earlier version of this paper. The financial support of GNAMPA INdAM 2020 projects "Processi stocastici quantistici e applicazioni" and "Evoluzioni markoviane quantistiche" is gratefully acknowledged. 


\section{References}

[1] L. Accardi, F. Fagnola, and R. Quezada, "On three new principles in non-equilibrium statistical mechanics and Markov semigroups of weak coupling limit type", Infin. Dimens. Anal. Quantum Probab. Relat. Top., vol. 19, no. 2, 1650009, 2016. DOI: 10.1142/S0219025716500090.

[2] L. Accardi, Y. G. Lu, and I. V. Volovich, Quantum theory and its stochastic limit, SpringerVerlag, Berlin, 2002.

[3] I. Ya. Aref'eva, I. V. Volovich, and S. V. Kozyrev, "Stochastic Limit Method and Interference in Quantum Many Particle Systems", Teor. Mat. Fiz., vol. 185, pp. 388-408, 2015. DOI: 10.1007/s11232-015-0296-9

[4] G. Basile, and S. Olla, "Energy diffusion in harmonic system with conservative noise", J. Stat. Phys., vol. 155, pp. 1126-1142, 2014. DOI: 10.1007/s10955-013-0908-4

[5] F. Benatti, R. Floreanini, and L. Memarzadeh, "Bath assisted transport in a three-site spin chain: global vs local approach", Phys. Rev. A, vol. 102, 042219-1-042219-14, 2020. DOI: 10.1103/PhysRevA.102.042219

[6] G. Benenti, G. Casati, T. Prosen, and D. Rossini, "Negative differential conductivity in far-from-equilibrium quantum spin chains", EPL, vol. 85, 37001, 2009. DOI: 10.1209/0295$5075 / 85 / 37001$

[7] C. Bernardin, and S. Olla, "Fourier's Law for a Microscopic Model of Heat Conduction", J. Stat. Phys., vol. 121, pp. 271-289, 2005. DOI: 10.1007/s10955-005-7578-9

[8] R. Carbone, E. Sasso, and V. Umanità, "Structure of generic quantum Markov semigroup", Infin. Dimens. Anal. Quantum Probab. Relat. Top., vol. 20, no. 2, 1750012, 2017. DOI: 10.1142/S0219025717500126

[9] J. Dereziński, and W. Roeck, "Extended Weak Coupling Limit for Pauli-Fierz Operators", Comm. Math. Phys., vol. 279, pp. 1-30, 2008. DOI: 10.1007/s00220-008-0419-3

[10] J. Dereziński, W. Roeck, and C. Maes, "Fluctuations of quantum currents and unravelings of master equations", J. Stat. Phys., vol. 131, pp. 341-356, 2008. DOI: 10.1007/s10955-008$9500-8$

[11] J. Deschamps, F. Fagnola, E. Sasso, and V. Umanità, "Structure of uniformly continuous quantum Markov semigroups", Rev. Math. Phys., vol. 28, no. 1, 1650003, 2016. DOI: 10.1142/S0129055X16500033 
[12] A. Dhar, and H. Spohn, "Fourier's law based on microscopic dynamics", C. R. Phys., vol. 20, pp. 393-401, 2019. DOI: 10.1016/j.crhy.2019.08.004

[13] E. B. Davies, "Markovian Master Equations", Comm. Math. Phys., vol. 39, pp. 91-110, 1974. projecteuclid.org/euclid.cmp/1103860160

[14] G. S. Engel, T. R. Calhoun, E. L. Read, T. -K. Ahn, T. Mancal, Y. -C. Cheng, R. E. Blankenship, and G. R. Fleming, "Evidence for Wavelike Energy Transfer Through Quantum Coherence in Photosynthetic Systems", Nature, vol. 446, pp. 782-786, 2007. DOI: 10.1038/nature05678

[15] F. Fagnola, and R. Rebolledo, "Entropy Production for Quantum Markov Semigroups", Commun. Math. Phys., vol. 335, pp. 547-570, 2015. DOI: 10.1007/s00220-015-2320-1

[16] F. Fagnola, and R. Rebolledo, "Entropy production and detailed balance for a class of quantum Markov semigroups", Open Syst. Inf. Dyn., vol. 22, no. 3, 1550013, 2015. DOI: $10.1142 / \mathrm{S} 1230161215500134$

[17] V. Gorini, A. Kossakowski, and E.C.G. Sudarshan, "Completely positive dynamical semigroups of $N$-level systems", J. Math. Phys., vol. 17, pp. 821-825, 1976. DOI: $10.1063 / 1.522979$

[18] J. M. Horowitz, and J. M. R. Parrondo, "Entropy production along nonequilibrium quantum jump trajectories", New J. Phys. vol. 15, 085028, 2013. DOI: 10.1088/1367-2630/15/8/085028

[19] V. Jakšić, C.-A. Pillet, and M. Westrich, "Entropic Fluctuations of Quantum Dynamical Semigroups". J. Stat. Phys., vol. 154, pp. 153-187, 2014. DOI: 10.1007/s10955-013-0826-5

[20] D. Karevski, and T. Platini, "Quantum nonequilibrium steady states induced by repeated interactions". Phys. Rev. Lett., vol. 102, 207207-1-20207-4, 2009, DOI: 10.1103/PhysRevLett.102.207207

[21] E. Langmann, J. L. Lebowitz, V. Mastropietro, and P. Moosavi, "Steady States and Universal Conductance in a Quenched Luttinger Model", Commun. Math. Phys., vol. 349, pp. 551-582, 2017. DOI: $10.1007 / \mathrm{s} 00220-016-2631-\mathrm{x}$

[22] G. Lindblad, "On the Generators of Quantum Dynamical Semigroups", Commun. Math. Phys., vol. 48, pp. 119-130, 1976. DOI: 10.1007/BF01608499

[23] M. Ohya, and D. Petz Quantum Entropy and its Use. Springer-Verlag, Berlin, 1993.

[24] G. D. Scholes, G. R. Fleming, A. Olaya-Castro, and R. van Grondelle, "Lessons from Nature about Solar Light Harvesting", Nature Chem., vol. 3, pp 763-774, 2011. DOI: doi.org/10.1038/nchem.1145 
[25] H. Spohn, and J. L. Lebowitz, "Irreversible Thermodynamics for Quantum Systems Weakly Coupled to Thermal Reservoirs". In, Advances in Chemical Physics, S.A. Rice (Ed.) pp. 109-142, 1978. DOI: doi.org/10.1002/9780470142578.ch2

[26] A. S. Trusheckin, "On the General Definition of the Production of Entropy in Open Markov Quantum Systems", J. Math. Sci. (N.Y.), vol. 241, pp. 191-209, 2019. DOI: 10.1007/s10958019-04417-4

[27] M. Vanicat, L. Zadnik, and T. Prosen, "Integrable trotterization: local conservation laws and boundary driving", Phys. Rev. Lett. vol.121, 030606-1-030606-6, 2018. DOI: 10.1103/PhysRevLett.121.030606 